\title{
Studying Tomorrow's Materials Today: Insights with Quantitative STEM, EELS
}

\author{
G. A. Botton ${ }^{1}$, M. Bugnet ${ }^{1}$, K.J. Dudeck ${ }^{1}$, N. Gauquelin ${ }^{1,2}$, H. Liu ${ }^{1}$, S. Prabhudev ${ }^{1}$, A. Scullion ${ }^{1}$, S. \\ Stambula $^{1}$, S.Y. Woo ${ }^{1}$, G.-Z. Zhu ${ }^{1}$, H.P.T. Nguyen ${ }^{3}$, Z. Mi $^{3}$ \\ 1. Department of Materials Science and Engineering, McMaster University, Hamilton, ON, Canada \\ 2. Now at EMAT, University of Antwerp, Antwerp, Belgium \\ ${ }^{3 .}$ Department of Electrical and Computer Engineering, McGill University, Montreal, QC, Canada
}

The development of aberration correctors for the scanning transmission electron microscope has revolutionized the field of electron microscopy and dramatically improved the analytical "toolkit" of materials scientists. In particular, when combined with electron energy loss spectroscopy (EELS), scanning transmission electron microscopy (STEM) makes it possible to detect compositional and spectroscopic changes at the atomic level that can be used to understand the structure, and ultimately the performance of materials. Here we present some examples of quantitative STEM and EELS as applied to the study of graphene-based materials, complex nanoparticles used in electrocatalysts for fuel cells, group III- nitride nanowires used for light emitting devices, and the defects generated in implanted Si.

An FEI Titan (80-300 Cubed) microscope, equipped with a monochromator and EELS spectrometer (Quantum 966) was used for this work. With this system, we have been able to image single Pt atoms on multilayer graphene nanosheets (GNS) and demonstrate that single Pt atoms are stabilized during atomic layer deposition on N-doped GNS. Quantitative analyses of STEM images show that the single atoms are located at GNS edge steps and that the doping strongly suppresses the growth of Pt clusters/particles (Figure 1) [1]. Similarly, quantitative STEM images have been used to detect atomic displacements on $\mathrm{PtFe}$ intermetallic core-shell nanoparticles that exhibit very high specific activity compared to pure $\mathrm{Pt}$ $[2,3]$. Not only is elemental mapping at the atomic scale possible, but the high beam current and fast spectrometers also allow the acquisition of these maps with large sampling of the nanostructure. This is illustrated in the study of PtRu nanocatalysts used in fuel cells where Ru core-Pt shell structures are very clearly mapped (Figure 2). In the same system, we will show that it is possible to obtain maps from the Ti $K$ edge $(\approx 4970 \mathrm{eV}$ loss $)$ and Pt $M 45$ edge $(\approx 2120 \mathrm{eV})$ using the dual-EELS capabilities of the spectrometer. Further studies with EELS demonstrate the detection of valence changes and mapping of valence in Li-ion battery materials.

Beyond the "simple" deduction of the distribution of elements in nanostructures from maps, quantification is essential to understand the detailed structure of defects and correlate compositional measurement with, for instance, the optical response of materials. The detailed quantification of the atomic position of a defect, in this case a so-called $\{311\}$ defect [4] generated by the implantation of ions in $\mathrm{Si}[4,5]$ shows that an excellent agreement is obtained between the experimental atomic positions and molecular dynamics simulations (Figure 3) [4] with an accuracy of better than $0.05 \mathrm{~nm}$ for more than 100 atomic columns. Similarly, quantitative analysis of SiGe alloys deposited on Si has allowed us to deduce compositional fluctuations and interdiffusion in proximity of interfaces with the substrate [6]. A quantitative analysis with EELS is also shown for the measurement of the composition of InGaN quantum dots in GaN nanowires. Here, through reference spectra, we show that the composition of the $\mathrm{In}_{\mathrm{x}} \mathrm{Ga}_{1-\mathrm{x}} \mathrm{N}$ dots is non-uniform between different dots and varies from wire to wire (Figure 4). These deductions have been further supported with emission wavelengths measured from cathodoluminescence in STEM and photoluminescence measurements [7,8]. 


\section{References:}

[1] S. Stambula et al., Journal of Physical Chemistry C, on-line (2014), DOI:

10.1021/jp408979h

[2] S. Prabhudev et al., ACS Nano 7, 6103-6110, (2013)

[3] M.C.Y. Chan et al, Nanoscale 4 (22), 7273-7279, (2012)

[4] K.J. Dudeck et al., Physical Review Letters, Physical Review Letters, 110, 166102 (2013)

[5] K.J. Dudeck et al., Semiconductor Science and Technology, 28, 125012, (2013) [6] G.

Radtke et al., Physical Review B 87, 205309, (2013) [7] S.Y. Woo et al., to be submitted

[8] The authors are grateful to NSERC for supporting this research. The microscopy was carried out at the Canadian Centre for Electron Microscopy, a National facility supported by NSERC and McMaster. We are grateful to Paolo Longo (Gatan Inc.) for the help in setting up the Quantum 966 spectrometer.
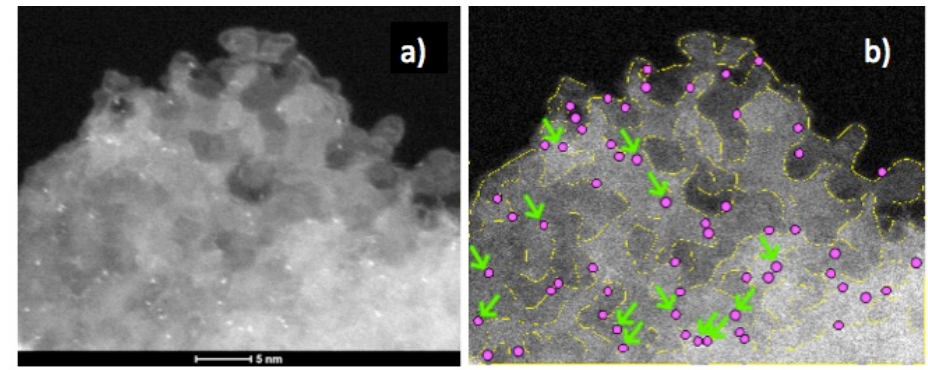

Figure 1, HAADF STEM image of single Pt atoms and clusters stabilized on N-doped GNS. Raw signals (a), edges and atoms detected (b). Green arrows point to the few Pt atoms (pink) stabilized on GNS terraces (edges labeled in yellow) [1].
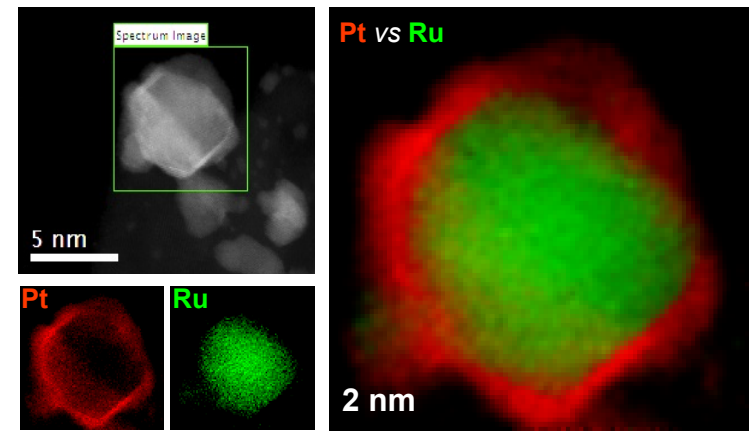

Figure 2, HAADF STEM image of PtRu nanoparticles used for fuel cell catalysts. $\mathrm{Pt}$ $M_{45}$ edge $(2122 \mathrm{eV})$ map in red, $\mathrm{Ru} L_{23}$ edge map $(2833 \mathrm{eV})$ in green.
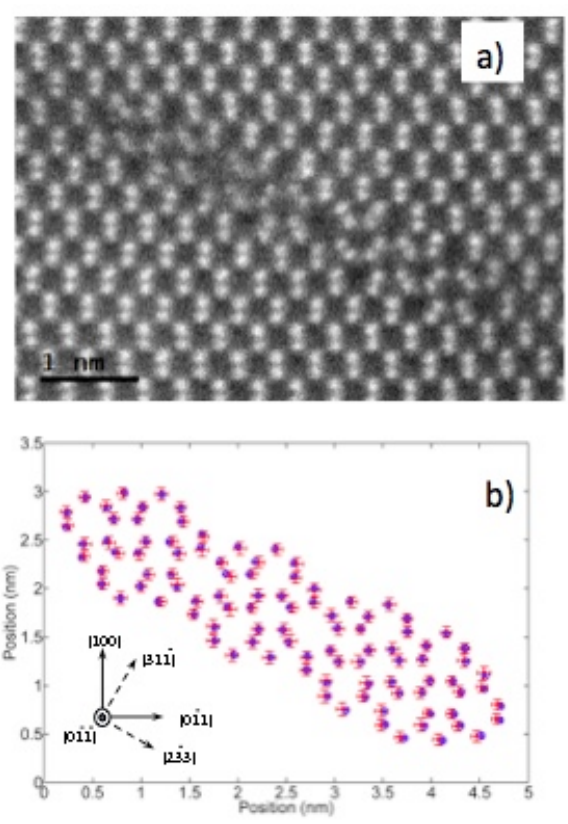

Figure 3, HAADF STEM image of a $\{311\}$ defect in $\mathrm{Si}$ (a) and the deduced atomic positions (crosses) in (b) with the expected atomic positions deduced by molecular dynamics calculations [4].

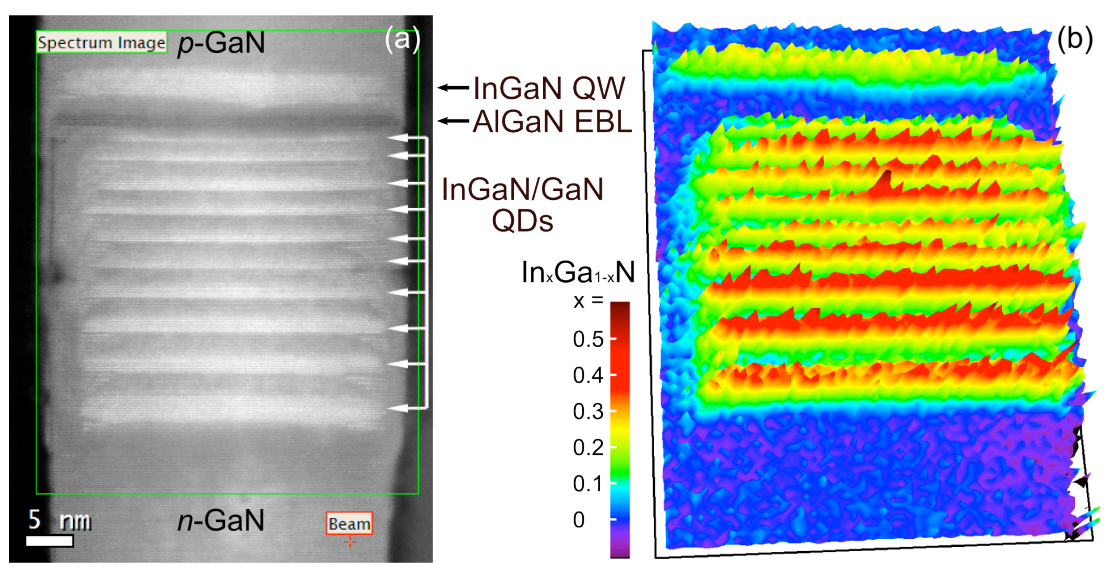

Figure 4, HAADF STEM image (a) showing the structure of the $\left(\operatorname{In}_{\mathrm{x}} \mathrm{Ga}_{1-\mathrm{x}} \mathrm{N}\right)$ dots in a GaN nano wire. Quantitative EELS map of the In composition in the nanowire showing the distribution of In within the dots and the variation of their composition. 